\title{
A FACTORIZATION-BASED PROJECTIVE RECONSTRUCTION ALGORITHM WITH CIRCULAR MOTION CONSTRAINT
}

\author{
Y. Li, W. K. Tang and Y. S. Hung \\ Department of Electrical and Electronic Engineering \\ The University of Hong Kong \\ Pokfulam Road, Hong Kong \\ \{yanli,wktang,yshung\}@eee.hku.hk
}

\begin{abstract}
In this paper, we propose a projective reconstruction algorithm for a circular motion image sequence. We first formulate the circular motion constraint in the Euclidean frame, and then deduce its expression in a projective frame. The circular motion constraint is gradually enforced during the iterations of a projective reconstruction. This approach can be used to deal with both constant and varying intrinsic parameters. Experimental results for synthetic and real data are presented to illustrate the performance and improvements of our approach over methods based on general motion.
\end{abstract}

\section{INTRODUCTION}

Circular motion image sequence is obtained when images are captured by a camera on a rotating arm or a fixed camera pointing towards an rotating object. Constructing 3D models from these special configurations has attracted a lot of attentions $[5,2,3]$.To makc use of the huge $2 \mathrm{D}$ point correspondences, Niem and Buschmann [5] provided a system for constructing 3D models from well-controlled circular motion (i.e., cameras are calibrated and the exact camera positions are measured). Fitzgibbon et al. [2] proposed to reduce the constraints to unknown constant intrinsic parameters over all the views. In [2], the reconstruction ambiguity for circular motion image sequence is discussed. However, the problem of varying intrinsic parameters for auto-focusing and zooming cameras is not addressed. Tang and Hung [6] proposed a factorization-based method by solving a sequence of weighted least-squares (WLS) problems to achieve minimization of $2 \mathrm{D}$ reprojection error.

In this paper, we propose a factorization-based method for projective reconstruction for a circular motion image sequence. The condition of constant intrinsic parameters [2] is relaxed to varying intrinsic parameters. We will give a formulation of the circular motion constraint in both the Euclidean and projective frames. During the iterative process in projective reconstruction, the constraint is enforced gradually.

The paper is organized as follows. The projective reconstruction problem is formulated in Section 2. Some background on the factorization-based method for projective reconstruction is given in Section 3. The theory of circular motion constraint is given in

The work described in this paper is partially supported by a grant from the Research Grant Council of the Hong Kong Special Administrative Region, China (Project No. HKU 7058/02E) and partially supported by CRCG of the University of Hong Kong.
Section 4. In Section 5, we formulate the problem of projective reconstruction with circular motion constraint. In Section 6, the case of constant intrinsic parameters is shown to be a special case of the varying parameter problem. The generalized algorithm is given in Section 7. Experimental results for synthetic and real data are presented in Section 8 to illustrate the performance and improvements of our approach over methods based on general motion. Concluding remarks are given in Section 9.

Notation: The Hadamard product of two matrices $A=\left[a_{i j}\right]$ and $B=\left[b_{i j}\right]$ of the same size is denoted

$$
A \quad B=\left[a_{i j} b_{i j}\right]
$$

\section{PROBLEM FORMULATION}

Consider multiple cameras with projection matrices $P_{i}$ viewing a set of 3D points $X_{j}$ captured as image points $x_{i j}$ on the image planes of the cameras as

$$
\lambda_{i j} x_{i j}=P_{i} X_{j}
$$

where $\lambda_{i j}$ is the projective depth for $x_{i j}$. For a known circular motion image sequence, the $i^{\text {th }}$ view is rotated around the $Z$-axis with a known angle $\theta_{i}$ w.r.t. the first view. Assuming that the projection matrix of the first view in Euclidean frame is $K_{1}\left[R_{1} \mid t_{1}\right]$ with $\theta_{1}=0$, the problem of projective reconstruction with known circular motion constraint is formulated as

$$
\min _{P_{i}, X_{j}} \sum_{i, j}\left|x_{i j}-\frac{1}{P_{i}^{3} X_{j}} P_{i} X_{j}\right|_{2}^{2}
$$

subject to $P_{i} H=\beta_{i} K_{i}\left[R_{1} \mid t_{1}\right] R_{z}\left(\theta_{i}\right)$

where $H \in \Re^{4 \times 4}$ is the projective transformation to upgrade the reconstructed projective frame to the Euclidean frame, $\beta_{i}$ is the scaling factor for the $i^{\text {th }}$ camera, and $P_{i}^{3}$ is the $3^{\text {rd }}$ row of $P_{i}$.

\section{BACKGROUND}

\subsection{Factorization-based method for projective reconstruction}

In the factorization approach to projective reconstruction, a basic problem is to estimate the depths $\lambda_{i j}$ for the image points $x_{i j}=$ $\left[\begin{array}{lll}u_{i j} & v_{i j} & 1\end{array}\right]^{T}$ so that the scaled measurement matrix $\left[\lambda_{i j} x_{i j}\right]$ can be factorized as a rank-4 product $P X$, where $P$ is the joint projection 


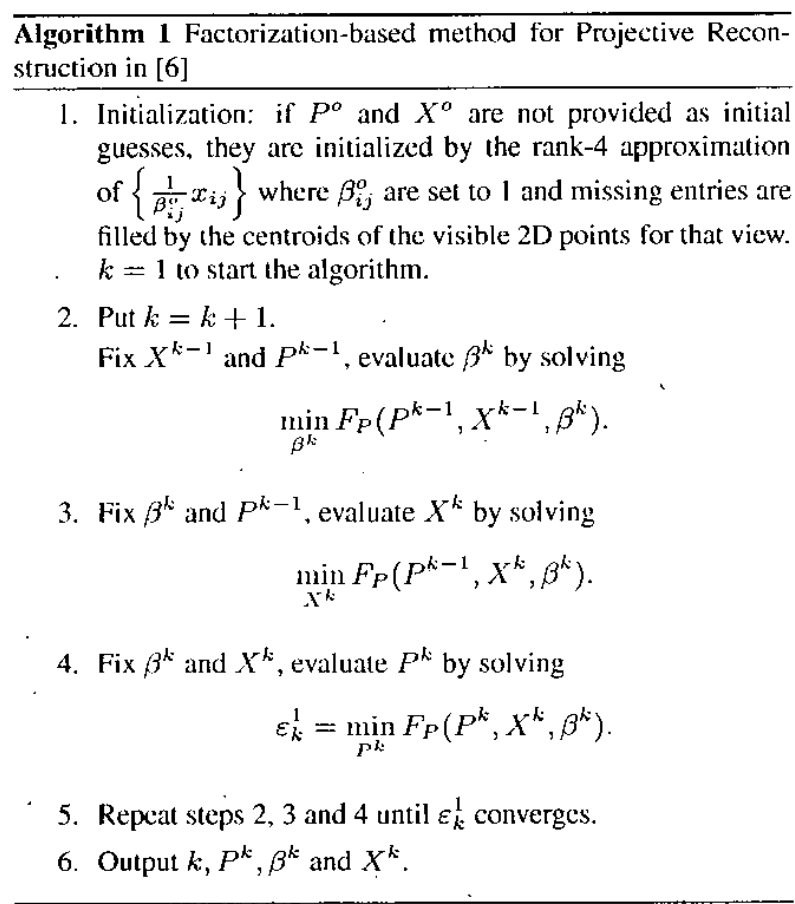

matrix and $X$ is a shape matrix containing the 3D points. Due to occlusion some points cannot be seen in all the views. Let us define the set $\mathcal{A}$ as

$$
\mathcal{A}=\left\{(i, j) \in \Re^{2} \mid X_{j} \text { is visible in the } i^{\text {th }} \text { view }\right\} .
$$

In [6], the cost function $F_{P}$ of the factorization-based method is proposed as

$$
F_{P}(P, X, \beta)=\sum_{(i, j) \in \mathcal{A}}\left\|\left[\begin{array}{c}
1 \\
1 \\
\gamma_{i j}
\end{array}\right]\left\{\left[\begin{array}{c}
u_{i j} \\
v_{i j} \\
1
\end{array}\right]-\beta_{i j} P_{i} X_{j}\right\}\right\|^{2}
$$

where $F_{P}(P, X, \beta)$ is the cost function of the factorization-based method, $\beta_{i j}=\frac{1}{\lambda_{i j}}$ and $\gamma_{i j}$ is the weighting factor for the 2D point $x_{i j}$ and usually chosen as $\max \left(\left|u_{i j}\right|,\left|v_{i j}\right|\right)$. A sequence of weighted linear least-squares (WLS) problems is formulated iteratively on $F_{P}(P, X, \beta)$ for solving one of $P, X$ and $\lambda$ as free parameters while fixing the other two parameters, as given in Algorithm 1 .

\subsection{Reconstruction Ambiguity}

It has been shown in [2] that for a camera with fixed intrinsic parameters undergoing known circular motion, if the first camera centre is at position $T=\left[\begin{array}{lll}t & 0 & 0\end{array}\right]^{T}$ and the camera is rotated around the $Z$-axis of the world coordinate system, the projection matrices $P_{i}$ in the Euclidean frame for the $i^{\text {th }}$ view can be written as

$$
P_{i}=M[I \mid T] R_{z}\left(\theta_{i}\right)
$$

where $M$ is a homography including the fixed intrinsic parameters $K$ and a rotation $R_{0}$ of the camera about its centre: $M=K R_{0}$, $I$ is the $3 \times 3$ identity matrix, and $R_{z}\left(\theta_{i}\right)$ is the rotation matrix of rotating by angle $\theta_{i}$ about the $Z$-axis. It is proved in [2] that if a reconstructed projection matrix $P_{i}^{\prime}=P_{i} H$ is determined with a set of cameras of the form (4), then $H$ can be parameterized as

$$
H=\left[\begin{array}{llll}
1 & 0 & 0 & 0 \\
0 & 1 & 0 & 0 \\
0 & 0 & \alpha & 0 \\
0 & 0 & \beta & 1
\end{array}\right]
$$

Hence, $P_{i}^{\prime}$ can be expanded as

$$
P_{i}^{\prime}=M[I \mid T] R_{z}\left(\theta_{i}\right) H .
$$

The result of [2] is also valid for cameras with varying intrinsic parameters $K_{i}$. That is for a camera with varying intrinsic parameters undergoing known circular motion, $M$ is replaced by $M_{i}=K_{i} R_{0}$ for all cameras in (4). Due to the special form of $H$, (5) can be rewritten as

$$
P_{i}^{\prime}=M_{i}[I \mid T] H R_{z}\left(O_{i}\right) .
$$

\section{CIRCULAR MOTION CONSTRAINT FOR PROJECTIVE RECONSTRUCTION}

It will be helpful in the following sections to consider that the object is fixed and that the camera rotates about a fixed axis around the object. The world coordinate system is chosen as in Section 3.2 .

For controlled circular motion, we may assume:

1. The camera centres lie on a circle in the motion plane.

2. The rotation angles $\theta_{i}$ between the $i^{t h}$ view and the first view are known.

Under the above assumptions, we can deduce that in the Euclidean frame the camera centre $O_{i}$ for the $i^{\text {th }}$ view satisfies the following equation

$$
O_{i}=R_{z}^{T}\left(\theta_{i}\right) O_{1}
$$

where $O_{1}$ is the camera centre of the first view. (7) will be referred to as the circular motion constraint. Our purpose is to find a projective reconstruction method which ensures that the camera centres satisfy $(7)$ in some Euclidean frame.

It has been shown in Section 3.2 that the projection matrix can be expressed as (6). For any $3 \times 3$ non-singular matrix $D$, we can insert $D D^{-1}$ between $K_{i}$ and $R_{0}[I \mid T]$ without changing the value of $P_{i}$. Denote $R_{0}[I \mid T]$ as $P_{0}$, the projection matrix can be written as

$$
P_{i}^{\prime}=K_{i} D D^{-1} P_{0} H R_{z}\left(\theta_{i}\right)=A_{i} B R_{z}\left(\theta_{i}\right)
$$

where $A_{i}=K_{i} D$ is a $3 \times 3$ non-singular matrix, $B=D^{-1} P_{0} H$ is a $3 \times 4$ matrix which remains unchanged for each camera. If we constrain $D$ to be an upper triangular matrix, $A_{i}$ is also an upper triangular matrix. The projective ambiguity now exists in the part of $A_{i} B$ instead of the whole projection matrix. The projection matrix $P_{i}^{\prime}$ can be upgraded to $P_{i}$ in the Euclidean frame as:

$$
P_{i}=P_{i}^{\prime} H^{-1}=A_{i} B H^{-1} R_{z}\left(\theta_{i}\right) .
$$

Since the camera centre can be calculated as the right null vector of its projection matrix [1] and $A_{i}$ is a $3 \times 3$ non-singular matrix, $C_{i}$ can be calculated as $C_{i}=\operatorname{null}\left(B H^{-1} R_{z}\left(\theta_{i}\right)\right)$. Hence, we have

$$
C_{i}=R_{z}^{T}\left(\theta_{i}\right) C_{1}
$$

(10) shows that if we can find a projective reconstruction with the form (8), we can ensure that the camera centres satisfy the circular motion constraint in the Euclidean frame. 


\section{A FACTORIZATION-BASED PROJECTIVE RECONSTRUCTION ALGORITHM WITH CIRCULAR MOTION CONSTRAINT}

To enforce the projection matrices to have the form (8), let us introduce the cost function,

$$
\begin{aligned}
& F_{C M}\left(A_{i}, B, X, \beta\right)= \\
& \sum_{(i, j) \in \mathcal{A}}\left\|\left[\begin{array}{c}
1 \\
1 \\
\gamma_{i j}
\end{array}\right]\left\{\left[\begin{array}{c}
u_{i j} \\
v_{i j} \\
1
\end{array}\right]-\beta_{i j} A_{i} B R_{z}\left(\theta_{i}\right) X_{j}\right\}\right\|^{2}
\end{aligned}
$$

where $A_{i}$ is an upper triangular matrix.

\subsection{From general motion to circular motion}

Given a reconstructed projective frame from Section 3.1, the camera centres can be expressed as: $O_{i}=\operatorname{null}\left(P_{i}\right), \quad i=1, \ldots, n$. Obviously, in the projective frame the camera centres do not satisfy (7) and $P_{i}$ does not have the form of (8). But there exists a projective transformation $H_{1}$ which will transform $O_{i}$ into a frame where (7) is satisfied. The next theorem shows that under such a transformation, the projection matrices will have the form (8).

Theorem 1 If we can find a $4 \times 4$ non-singular matrix $H_{c}$ to transform the projective frame so that the camera centre $O_{i}^{\prime}$ and the principle plane $p_{i}^{\prime 3}$ of the $i^{\text {th }}$ camera in the new projective frame should satisfy the following equations

$$
\begin{aligned}
O_{i}^{\prime} & =R_{z}^{T}\left(\theta_{i}\right) O_{1}^{\prime} \\
p_{i}^{\prime 3 T} & =p_{1}^{\prime 3 T} R_{z}\left(\theta_{i}\right)
\end{aligned}
$$

where $p_{i}^{\prime 3 T}$ is the third row of the $i^{\text {th }}$ camera matrix $P_{i}^{\prime}$. Both $O_{i}^{\prime}$ and $p_{i}^{\prime 3 T}$ are scaled so their last entries are equal to one. Then the projection matrices $P_{i}^{\prime}$ in the new projective frame can be expressed as

$$
P_{i}^{\prime}=P_{i} H_{c}^{-1}=K_{i} P_{0} H R_{z}\left(\theta_{i}\right),
$$

i.e. the projection matrices are in form of $(8)$.

Due to the limit of space, we omit the proof for Theorem 1.

\subsection{Decomposition of Projection matrices}

According to Theorem 1, after transformation with the matrix $H_{1}$ the new projection matrices $P_{i}^{\prime}$ can be written as

$$
P_{i}^{\prime}=A_{i} B R_{z}\left(\theta_{i}\right) \text {. }
$$

From (12), we have $P_{i}^{\prime} R_{z}^{T}\left(\theta_{i}\right)=A_{i} B$. Since all the row vectors of the matrix $A_{i} B$ are linear combinations of the three independent row vectors of $B$, if we stack all $P_{i}^{\prime} R_{z}^{T}\left(\theta_{i}\right)(i=1, \ldots, n)$ into one $3 n \times 4$ matrix $P$, the rank of $P$ should still be 3 . By means of Singular Value Decomposition, $P$ can be decomposed as $P=U S V^{T} . B$ can be chosen as $B=V_{3}^{T}$, where $V_{3}$ contains the first three rows of $V$.

\section{ALGORITHM FOR CONSTANT INTRINSIC PARAMETERS}

When the camera intrinsic parameters $K_{i}$ are kept constant over all the views, it can be considered as a special case of our circular motion constraint. The above algorithm can be modified slightly to handle this scenario. The constant intrinsic parameters can be treated as $A_{i}=I, \forall i$. In this case there is no need to estimate $A_{i}$.

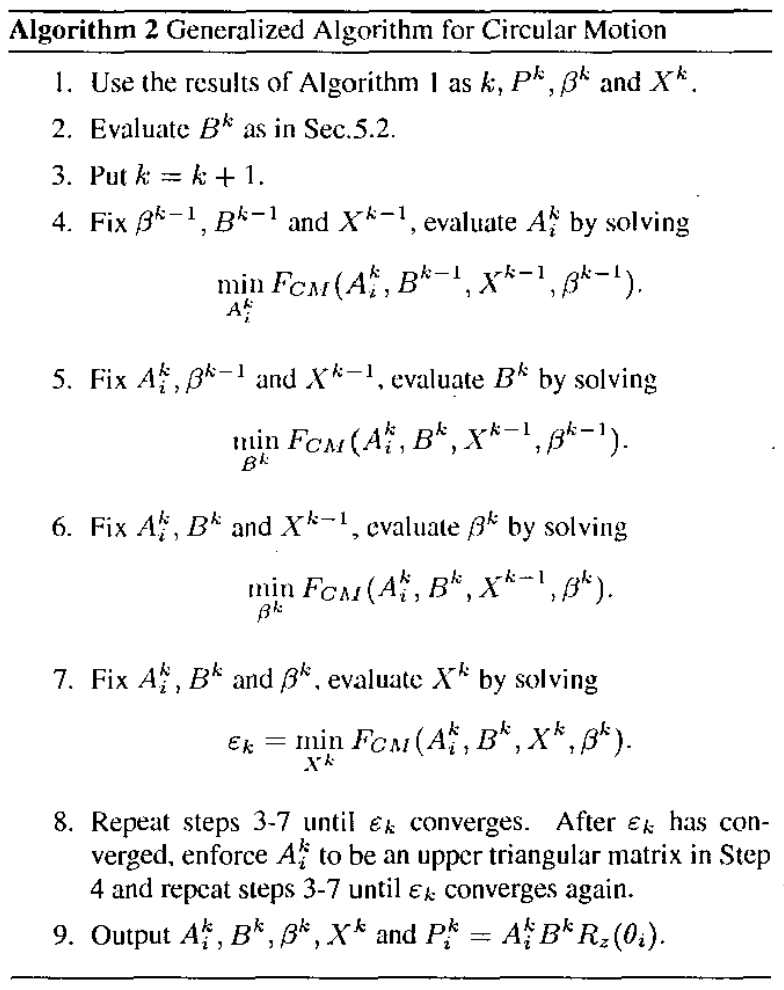

\section{THE GENERALIZED ALGORITHM}

The complete generalized algorithm for circular motion constraint with varying and constant intrinsic parameters is given in Algorithm 2. Since (11) is a quadrilinear (trilinear for the cases of constant intrinsic parameters) minimization problem in $A_{i}, B, X$ and $\beta,(11)$ is expressed as four different WLS problems where $A_{i}, B, X$ and $\beta$ are evaluated one by one iteratively while keeping the others unchanged. The superscript $k$ indicates that the variables are updated in the $k^{\text {th }}$ iteration. The constraint of $A_{i}$ being upper triangular matrices is enforced after the algorithm converges with $A_{i}$ being general $3 \times 3$ non-singular matrices. For the case of constant intrinsic parameters, Step 4 for estimating $A_{i}$ in the algorithm can be skipped.

\section{EXPERIMENTAL RESULTS}

\subsection{Synthetic Data}

In this simulation, we compare the results of our algorithm with the method given in [6]. The test scene consists of 30 points uniformly distributed within the unit sphere centered at the origin of the world coordinate system. The synthetic camera moving on the $x-y$ plane along a circular path of 7 meter radius captures 8 images. Gaussian noise with standard deviation ranging from 0.5 pixels to 4.5 pixels is added to the image points. The image size is about $1000 \times 1200$ pixels.

To measure the quality of the reconstruction, we calculate the 2D reprojection error and 3D residual of the Euclidean distances between the reconstructed scene points and their ground truth positions. The graphs show the median value over 8 trials. 


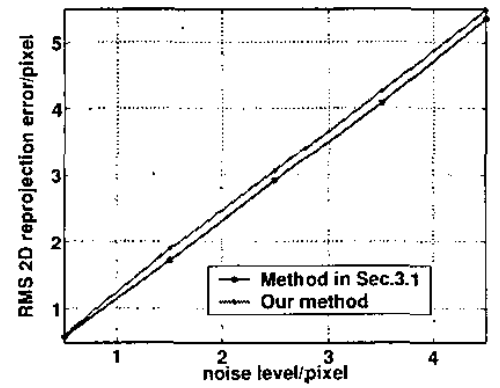

Fig. 1. 2D Reprojection Error

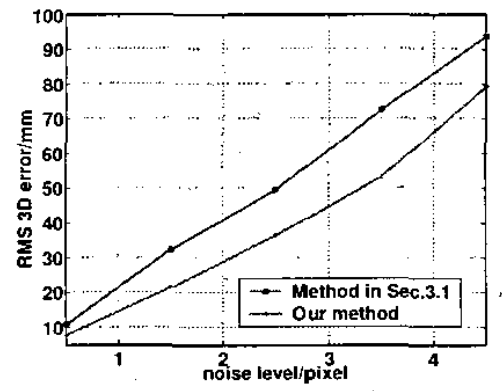

Fig. 2. 3D Error

\subsubsection{Performance on 2D Reprojection Error}

Fig. 1 shows the 2D reprojection error of the two algorithms against different noise levels. It is reasonable that the $2 \mathrm{D}$ reprojection error of our algorithm is slightly larger than the method in [6], because the degree of freedom is less.

\subsubsection{Performance on $3 D$ Error}

A transformation $H$ is computed by an optimization method to upgrade the reconstructed shape $X$ to the ground truth $M$ so that $M_{i}=H X_{j}$ has minimal 3D error. In Fig. 2, it can be seen that the reconstructed scene is closer to the ground truth under the circular motion constraint.

\subsection{Real Data}

The projective reconstruction algorithm is tested with a sequence of images taken by a static camera looking at a model house on a turntable. The intrinsic parameters of the camera are fixed while capturing the images so there is no need to estimate $A_{i}$. The

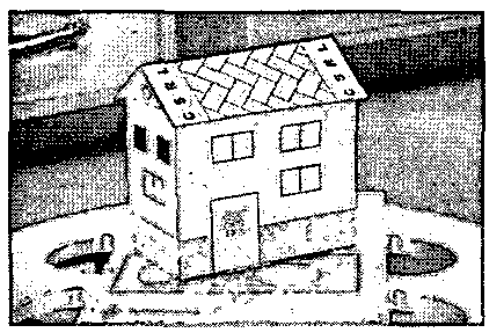

Fig. 3. An image for the circular motion image sequence

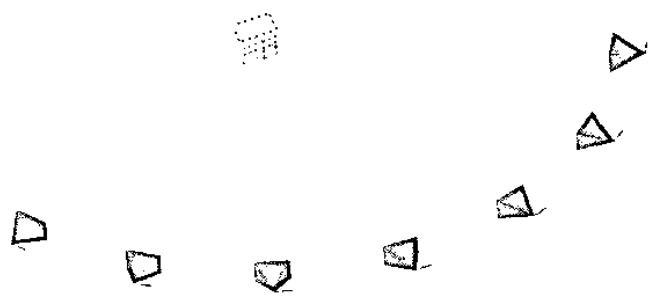

Fig. 4. A Reconstructed Scene

turntable is rotated by an angle $10^{\circ}$ incrementally for each image. A total of 7 images are taken and one of the images is shown in Fig. 3. The size of the images is $2000 \times 3000$ pixels. There are 33 corresponding points which are matched manually and visible to all the views. Our implementation of Algorithm 2 took 726 iterations to converge which required 273 seconds on a $3.4 \mathrm{GHz}$ PC Pentium-4 with IGB RAM. The RMS 2D reprojection error is 1.107 pixels. Fig. 4 shows the recovered the Euclidean structure and motion. The 3D points are shown and the cameras are represented by pyramids.

\section{CONCIUUSION}

In this paper, we propose a new projective reconstruction method for minimizing $2 \mathrm{D}$ reprojection error for a circular motion image sequence. This generalized algorithm can be applied to image sequences with varying or constant intrinsic parameters. With the circular motion constraint, the accuracy of reconstruction can be improved in terms of 3D error and the reconstructed projective frame can provide accurate results for the camera calibration methods relying on epipolar geometry (e.g., [4]).

\section{References}

[1] O. D. Faugeras. Three-Dimensional Computer Vision: A Geometric Viewpoint. The MIT Press, Cambridge, Mass., 1993.

[2] A. W. Fitzgibbon, G. Cross, and A. Zisserman. "Automatic 3D Model Construction for Turn-Table Sequences". In Proc. of SMILE Workshop on $3 D$ Structure from Multiple Images in Large Scale Environments, vol.LNCS-1506, pages 154-170, June 1998.

[3] P.R.S. Mendonça, K.-Y. K. Wong, and R. Cipolla. "Epipolar Geometry from Profiles under Circular Motion". IEEE Trans. Pattern Analysis \& Machine Intelligence, 23(6):604 616, June 2001.

[4] P.R.S. Mendonça and R. Cipolla. "A simple technique for self-calibration". In Int. Conf. on Computer Vision \& Pattern Recognition, volume I, pages 500-505, 1999.

[5] W. Niem and R. Buschmann. "Automatic Modelling of 3D Natural Objects from Multiple Views". In European Workshop on Combined Real and Synthetic Image Processing for Broadcast and Video Production, 1994.

[6] W. K. Tang and Y. S. Hung. "A Factorization-based method for Projective Reconstruction with minimization of 2-D reprojection errors". In Proc. of 24th DAGM 2002, vol. LNCS2449, pages 387-394, September 2002. 